\title{
Fuzzy tungsten in a magnetron sputtering device
}

DOI:

10.1016/j.jnucmat.2016.08.019

\section{Document Version}

Accepted author manuscript

Link to publication record in Manchester Research Explorer

\section{Citation for published version (APA):}

Petty, T. J., Khan, A., Heil, T., \& Bradley, J. W. (2016). Fuzzy tungsten in a magnetron sputtering device. Journal of Nuclear Materials, 480, 374-385. https://doi.org/10.1016/j.jnucmat.2016.08.019

\section{Published in:}

Journal of Nuclear Materials

\section{Citing this paper}

Please note that where the full-text provided on Manchester Research Explorer is the Author Accepted Manuscript or Proof version this may differ from the final Published version. If citing, it is advised that you check and use the publisher's definitive version.

\section{General rights}

Copyright and moral rights for the publications made accessible in the Research Explorer are retained by the authors and/or other copyright owners and it is a condition of accessing publications that users recognise and abide by the legal requirements associated with these rights.

\section{Takedown policy}

If you believe that this document breaches copyright please refer to the University of Manchester's Takedown Procedures [http://man.ac.uk/04Y6Bo] or contact uml.scholarlycommunications@manchester.ac.uk providing relevant details, so we can investigate your claim.

\section{OPEN ACCESS}




\title{
Fuzzy tungsten in a magnetron sputtering device
}

\author{
T.J. Petty ${ }^{\mathrm{a}}$, A. Khan ${ }^{\mathrm{b}}$, T. Heil ${ }^{\mathrm{c}}$, and J.W. Bradley ${ }^{\mathrm{a}}$ \\ ${ }^{a}$ Department of Electrical Engineering and Electronics, University of Liverpool, Brownlow \\ Hill, Liverpool, L69 3GJ, UK \\ b Pariser Building-G11, School of Mechanical, Aerospace and Civil Engineering, The \\ University of Manchester, Manchester, M13 9PL \\ ${ }^{\mathrm{c}}$ NiCaL, Block C Waterhouse Building, 1-3 Brownlow Street, Liverpool, L69 3GL
}

\begin{abstract}
Helium ion induced tungsten nanostructure (tungsten fuzz) has been studied in a magnetron sputtering device. Three parameters were varied, the fluence from $3.4 \times 10^{23}-3.0 \times 10^{24} \mathrm{~m}^{-2}$, the He ion energy from $25-70 \mathrm{eV}$, and the surface temperature from 900 - 1200 K. For each sample, SEM images were captured, and measurements of the fuzz layer thickness, surface roughness, reflectivity, and average structure widths are provided. A cross-over point from pre-fuzz to fully formed fuzz is found at $2.4 \pm 0.4 \times 10^{24} \mathrm{~m}^{-2}$, and a temperature of $1080 \pm 60 \mathrm{~K}$. No significant change was observed in the energy sweep. The fuzz is compared to low fluence fuzz created in the PISCES-A linear plasma device. Magnetron fuzz is less uniform than fuzz created by PISCES-A and with generally larger structure widths. The thicknesses of the magnetron samples follow the original $\Phi^{1 / 2}$ relation as opposed to the incubation fluence fit.

Keywords: Fuzzy tungsten, Magnetron, Helium
\end{abstract}

\section{Introduction}

Fuzzy tungsten is a phenomenon whereby a tungsten (W) sample is deformed from a mirror-finish to a black surface covered by a fibreform nano-structure, often referred to as 'fuzz'. This deformation occurs due to three conditions

Email address: j.w.bradley@liverpool.ac.uk (T.J. Petty ${ }^{\mathrm{a}}$, A. Khan ${ }^{\mathrm{b}}$, T. Heil ${ }^{\mathrm{c}}$, and J.W. Bradley ${ }^{\mathrm{a}}$ )

Preprint submitted to Journal of Nuclear Materials

June 13, 2016 
5 being fulfilled. A W sample must i) be bombarded by helium (He) ions for a sufficient amount of fluence (flux $\times$ time) $\left(\gtrsim 2.5 \times 10^{24} \mathrm{~m}^{-2}\right)$ [1, 2], ii) the $\mathrm{He}$ ions must have sufficient energy $(\gtrsim 20 \mathrm{eV})[3,4,5$, and iii) the $\mathrm{W}$ samples are of a sufficient temperature during bombardment ( $\gtrsim 1000 \mathrm{~K})$ [6, 5, 7].

In ITER, the next generation fusion reactor currently being constructed in 10 France, part of the divertor region is expected to have the same conditions for generating fuzz [8, 9, 10, 11, 12, 13. As such it is an active area of research. However, there is also interest outside of fusion power. Recently it has been used in the splitting of water molecules [14, and some authors expect that its high surface area could be harnessed as a catalyst [15. Also, due to its very low reflectivity, it could prove useful in the solar cell industry [16].

There has previously been some research studying the formation conditions of fuzz, with samples being exposed over a wide range of parameters [5, 4]. However, the region of low fluence $\left(<10^{25} \mathrm{~m}^{-2}\right)$ has been often overlooked, with little work on fuzz generation presented here. Iyyakkunnel et al. exposed sam20 ples in a magnetron sputtering device with fluences of $\sim 1 \times 10^{23} \mathrm{~m}^{-2}$ showing the early stages of fuzz formation [17. Work on the Nano-PSI device at DIFFER by El-Atwani et al. exposed samples with fluences of $5 \times 10^{20}-1 \times 10^{23} \mathrm{~m}^{-2}$, again showing the early signs of surface deformation, however, not quite creating fuzz yet [18. Woller et al. exposed two low fluence samples in a helicon plasma 25 source (DIONISOS) at fluences of $2.6 \times 10^{23}$ and $4.9 \times 10^{24} \mathrm{~m}^{-2}$, showing fuzz formation in the latter but not at the former [19]. Lastly, the lowest fluence samples found to be exposed in NADGIS was $6 \times 10^{24} \mathrm{~m}^{-2}$, also showing fuzz formation [5]. The low fluence work thus conducted in the literature seems to be showing a gap between $1 \times 10^{23}-1 \times 10^{25} \mathrm{~m}^{-2}$. While there have been exposures within this range, a dedicated parameter sweep is missing thus far. The present results cover the fluence from $3 \times 10^{23}-4 \times 10^{24} \mathrm{~m}^{-2}$ in a systematic approach to bridge the gap from early stages of surface modification to fully formed fuzz. This region is important to investigate as it contains the proposed incubation fluence, as introduced in 2. This is the proposed fluence of He ions necessary 35 before fuzz can begin to grow. 
The samples in the present article were exposed in a magnetron sputtering device, which provides maximum fluences of the order of $10^{24} \mathrm{~m}^{-2}$. Typically, fuzz made in the literature uses linear plasma devices (LPDs) with an exposure fluence of $10^{25}-10^{27} \mathrm{~m}^{-2}$. Due to the large fluxes used in LPDs when studying 40 the low fluence region, the errors on the fluence can be as large as $10^{25} \mathrm{~m}^{-2}$ [2]. As such, they are not suitable for studying fluences of the order of $10^{24}$ $\mathrm{m}^{-2}$ and below. Also, in LPDs, the sample is heated by the plasma, and as such the temperature of the sample is dependent on the plasma conditions. In a magnetron device the heating generated by the plasma is not sufficient for 45 fuzz to form, therefore a separate heating method is used, thereby liberating the sample temperature from the plasma conditions.

Magnetrons also present a significant difference to other low flux devices in that deposition is occurring onto the growing fuzz sample throughout the exposure. This presents a unique situation of growing fuzz whilst having $\mathrm{W}$ deposited on top of it. This may potentially emulate the situation in DEMO, the future fusion power plant, which will likely have $\mathrm{W}$ covering all of the plasma facing components [20]. Thus $\mathrm{W}$ could be eroded from the main wall of the tokamak and be deposited in the divertor region, the region where fuzz is most likely to occur. Also, due to elevated wall temperatures, fuzz could develop on 55 parts of the main first wall [21].

A recent paper showed that it is possible to create fuzz structure in a magnetron device similar to that found in LPDs 22 . The present work studies the formation conditions of fuzz generation in a magnetron device. There are three main parameters concerning fuzz formation; being He fluence, He ion energy, and $\mathrm{W}$ surface temperature, these were each swept in the magnetron revealing new insights into the formation conditions. These conditions are compared to previous work in the literature and discrepancies are discussed. 


\section{Experimental method}

For the plasma exposure a magnetron sputtering device was used [22]. A

65 was a V-Tech ${ }^{\mathrm{TM}} 150$ unbalanced magnetron sputtering source (Gencoa Ltd.) mounted on one side of the chamber. The samples were held by a substrate holder facing the target and positioned along its axis at a distance of $93 \mathrm{~mm}$. The samples were heated by electron bombardment from a heated filament pyrometer (Micro-Epsilon UK Ltd.).

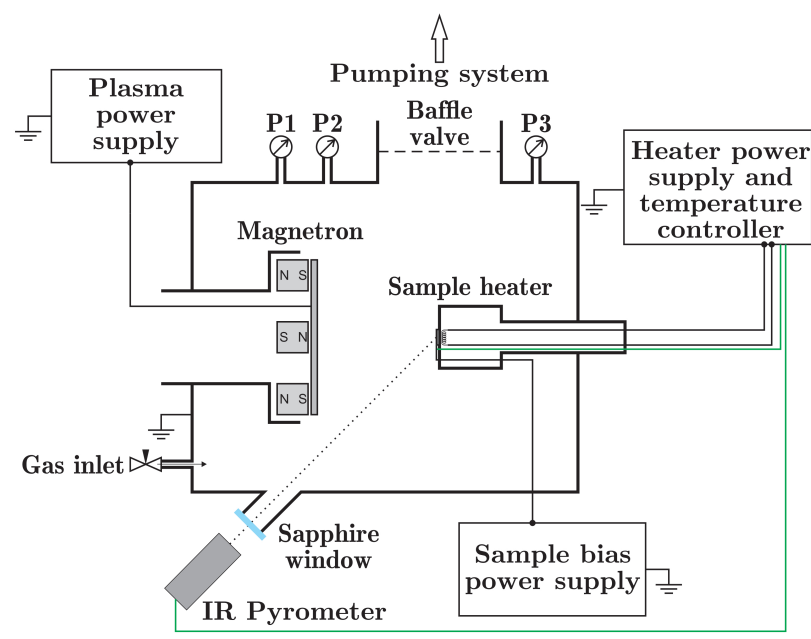

Figure 1: Schematic of the experimental rig.

In order to measure the plasma diagnostics, a special steel sample, insulated from the sample holder, was connected to a Langmuir probe acquisition box. Operating at $700 \mathrm{~W}$ with $5.3 \mathrm{~Pa} \mathrm{He}$ gas pressure [22, the plasma potential was measured at $-3.2 \pm 1 \mathrm{eV}$, and the He ion flux, which varied depending on the bias of the sample [23, 24, was of the order of $1 \times 10^{20} \mathrm{~m}^{-2} \mathrm{~s}^{-1}$.

The samples used in this study were $99.95 \%$ tungsten discs (Future Alloys) of $10 \mathrm{~mm}$ diameter and $1 \mathrm{~mm}$ thickness. They were prepared by first polishing with wet and dry sandpaper, followed by electro-polishing $(\mathrm{KOH})$ for $\sim 15$ minutes 
to a mirror finish. The emissivity of the samples was separately determined to be $0.26 \pm 0.02$.

The operating procedure began by first heating the sample to the desired temperature with He gas present (but with the plasma off at this stage). Once the temperature was reached, the plasma was generated, then the sample bias was applied, at which point the time of exposure was deemed to begin.

Throughout exposure, due to the nature of the magnetron device, sputtered $\mathrm{W}$ atoms from the target were deposited on the growing samples. Using a quartz crystal microbalance (QCM) (Maxtek), this deposition rate was separately measured to be $5.3 \pm 2.3 \mathrm{pms}^{-1}$ (or a depositing $\mathrm{W}$ flux of $3.3 \times 10^{17} \mathrm{~m}^{-2} \mathrm{~s}^{-1}$ ). The sputter threshold energy for He ions bombarding a W surface is $\sim 108 \mathrm{eV}$, however, the sputter yield for $\mathrm{W}$ impinging on $\mathrm{W}$ for $\sim 50 \mathrm{eV}$ is $1.9 \times 10^{-4}$ [25]. Therefore some sputtering of the growing samples will occur, but the flux of W atoms being sputtered from the sample is at least 4 orders of magnitude lower than the incoming flux. Also, magnetrons tend to have ionised metal flux fractions of $<10 \%$, so the effect of this sputtering has been neglected in the present study.

After exposure, samples were analysed using a variety of techniques. They were each imaged with an SEM (JEOL 7001) in order to provide images of the surface structure. The reflectivity of each sample was measured using a USB2000+ spectrometer connected by fibre optic cable to a DH-2000-BAL deuterium and halogen light source (Ocean Optics). The surface roughness of each sample was measured using a VK-X210 confocal microscope (Keyence). Lastly, the thickness of the fuzz layer of the samples was measured using a FEI Helios NanoLab 600i focused ion beam (FIB) of gallium atoms to first deposit a protective layer of platinum on top of the fuzz, then the beam (at a higher intensity) was used to mill out a trench. The fuzz cross-section could then be observed using the SEM component of the Nanolab. 


\section{Results}

Samples were exposed under a sweep of the three main parameters for fuzz

110

- $9 \mathrm{~h}$ in steps of $1 \mathrm{~h}$, whilst keeping the He ion energy fixed at $40 \mathrm{eV}$, the flux at $9.4 \times 10^{19} \mathrm{~m}^{-2} \mathrm{~s}^{-1}$, and the surface temperature at $1100 \mathrm{~K}$. The SEM images of the time sweep are shown in figure 2. Secondly the sample bias was swept, hence sweeping the bombarding He ion energies. This was done from 25 - 70 samples sitting generally above the line, apart from the one outlier at $4 \mathrm{~h}$, they 
all seem to follow a trend. The energy sweep does not show much variation, however, $60 \mathrm{eV}$ stands out with particularly wider error bars. This might be due to the many factors at play in this sweep, as the flux and the fluence are also changing between samples as well as the energy, hence it is difficult to draw a conclusion from this chart. Lastly the temperature sweep shows little variation until $1100 \mathrm{~K}$, where the increase in thickness undergoes the largest change seen in all the parameter steps, with the error bars significantly increasing too.

In figure 7 the roughness of every sample shown in the SEM images is presented. Roughness can be simply associated with fuzz formation, the thicker the fuzz, generally the rougher the surface, due to the nature of the fuzz structure. In the fluence sweep, there is no apparent change until $8 \mathrm{~h}$ when the roughness rises significantly. In the energy sweep there is an apparent rise up to $60 \mathrm{eV}$, followed by a decrease. Lastly for the temperature, the most drastic change is seen again between 1050 and $1100 \mathrm{~K}$, this is in line with the trend in thickness.

Finally in figure 8 the reflectivity of each sample was measured. The wavelength chosen for the figures was $632.8 \mathrm{~nm}$, fitting with work on reflectivity values by other authors 6 . It can be seen that these reflect the same information as the roughness curve. This is in part due to rougher surfaces having less reflectivity, therefore the two properties are interconnected. In fig. 8 a, showing the fluence sweep, a clean sample is shown at $0 \mathrm{~h}$ exposure, with a reflectivity of $81 \%$. The reflectivity gradually descends over time and appears to be plateauing at around $9 \mathrm{~h}$. In the energy sweep the reflectivity is generally low throughout, 160 with the lowest reflectivity of $3 \%$ recorded at $60 \mathrm{eV}$. For the temperature sweep, as was seen for the thickness and roughness curves, the most significant change is between 1050 and $1100 \mathrm{~K}$, and the lowest recorded reflectivity for all samples was $0.8 \%$ at $1200 \mathrm{~K}$.

Using the SEM images as presented in figures 2, 4 the widths of the structures were measured using the ImageJ software. In the lower fluence samples, where the streaks or ripples appear, the width of these ripples was measured. Also the globules widths were measured, though when it was not possible to define a width, the length across the whole globule was measured, often from several 
directions, to get an average structure size. Where tendrils appeared the widths were measured, but not the lengths. For each sample, 100-280 structures were measured and an average taken with $2 \times$ the standard deviation used for the error bars. The measurements for each sample in the parameter sweeps are shown in figure 9 . 


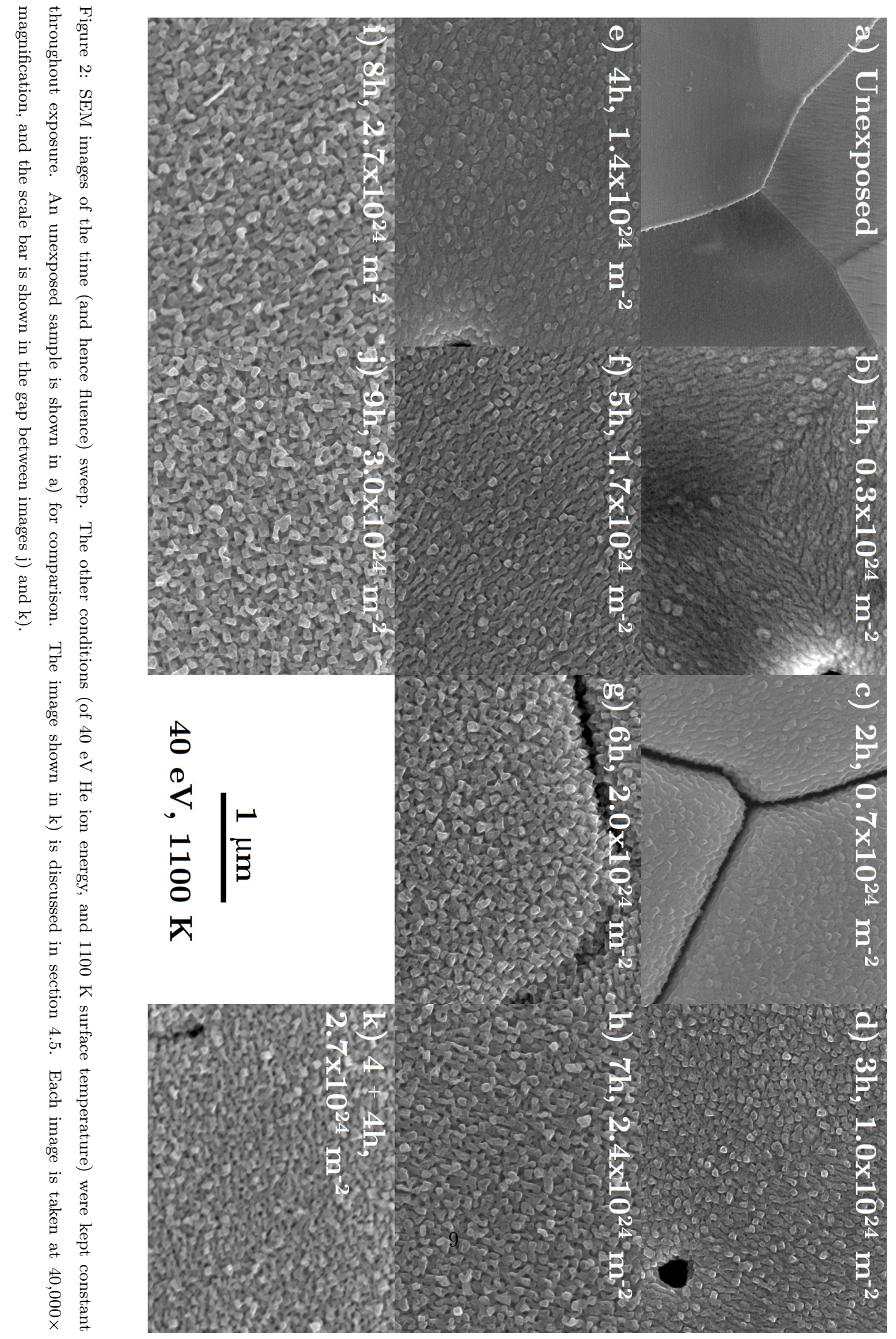




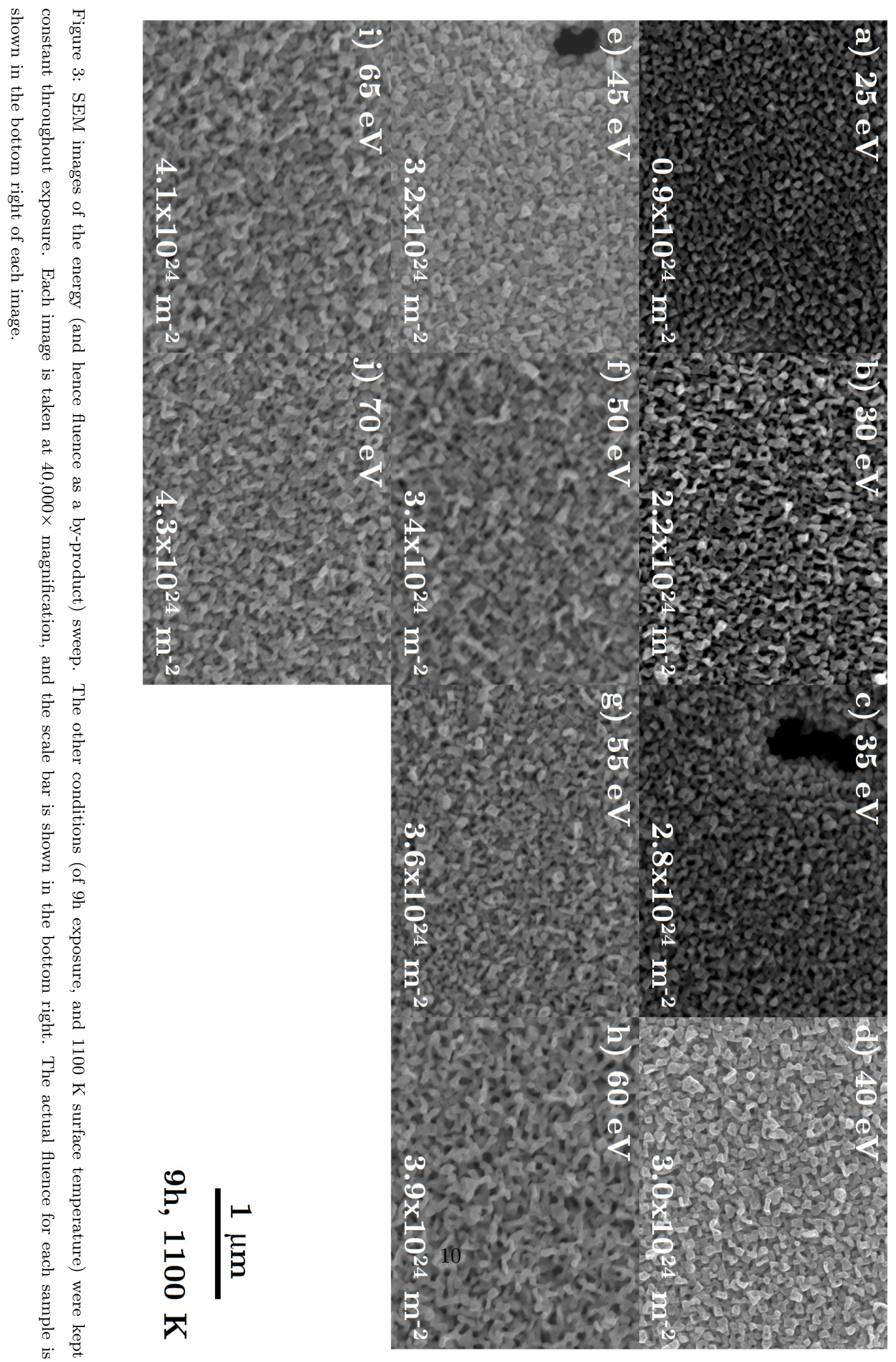




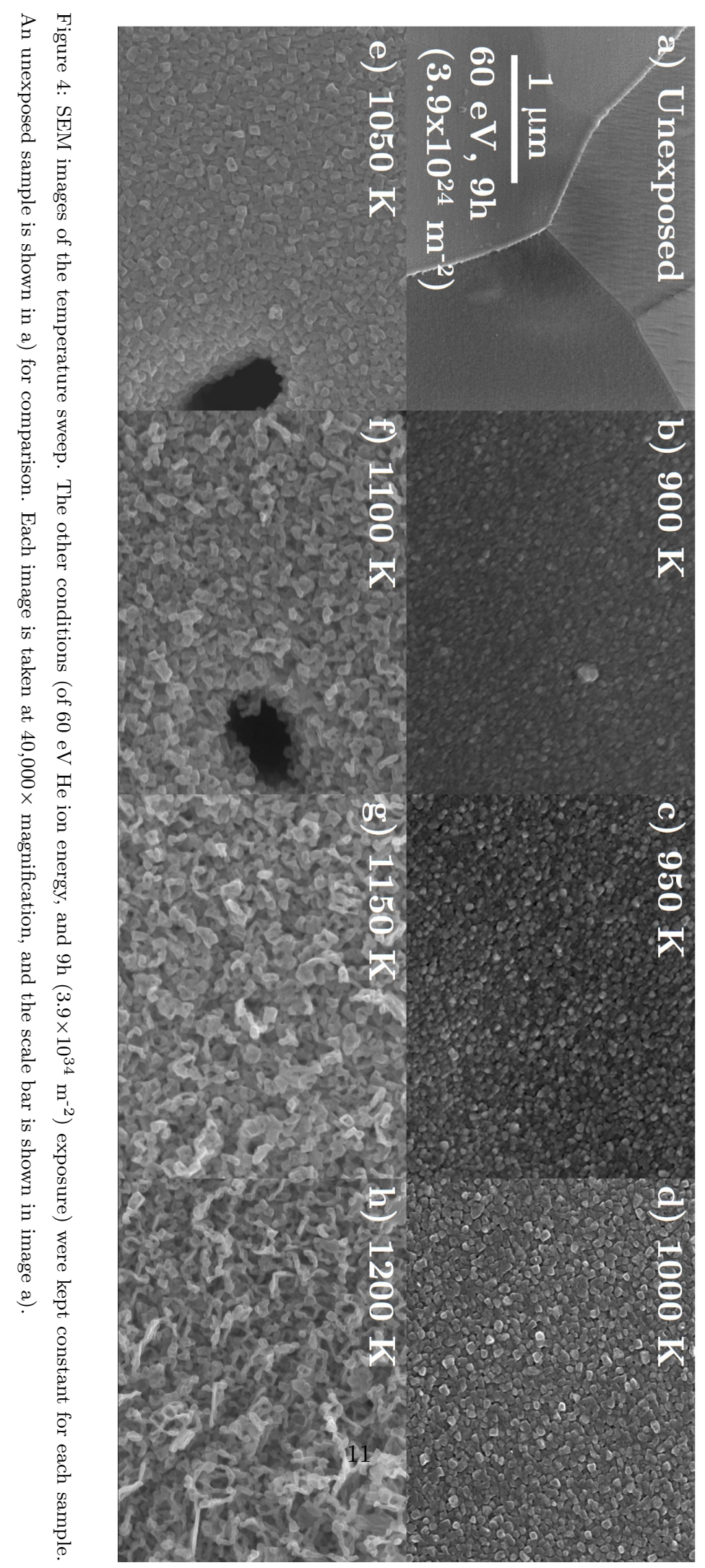




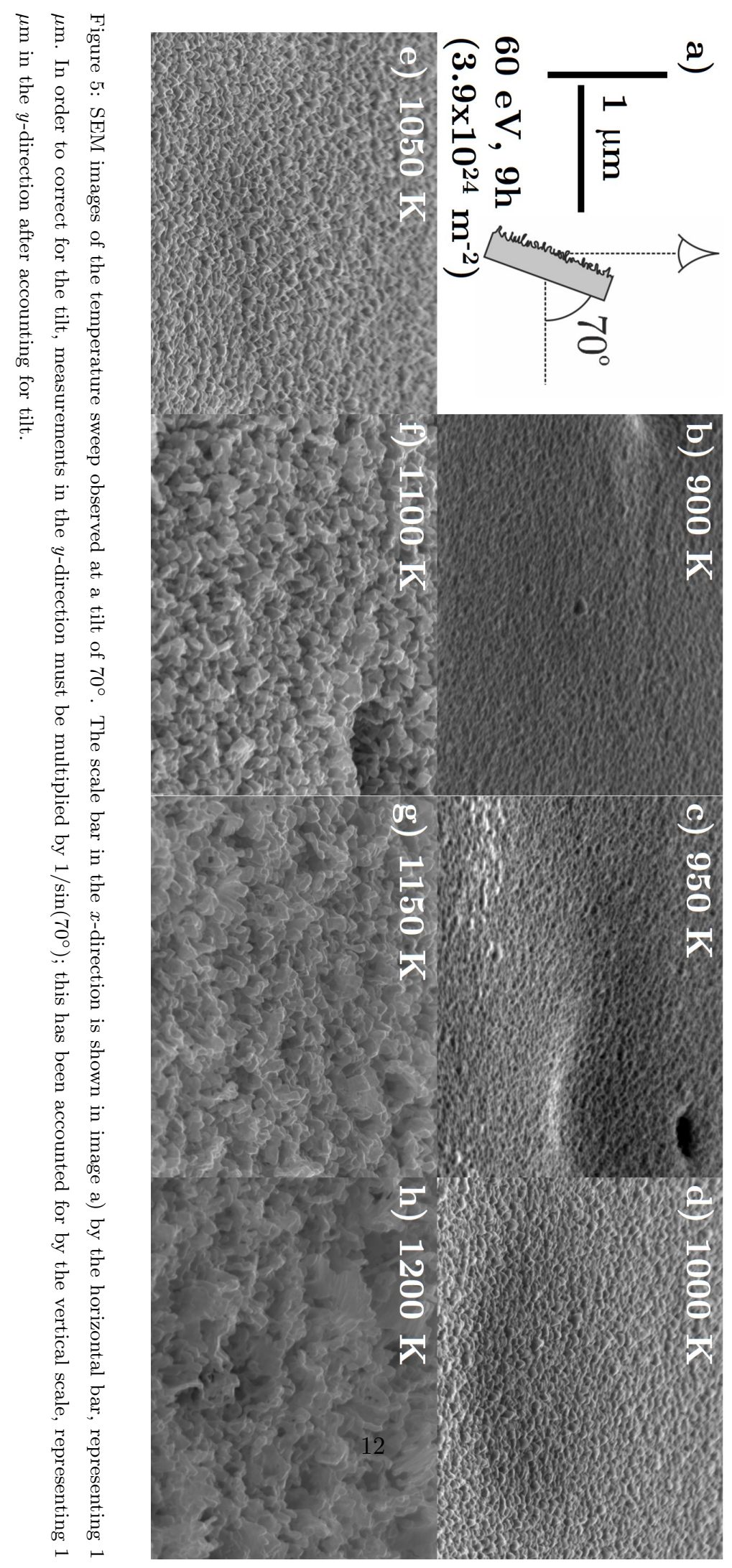




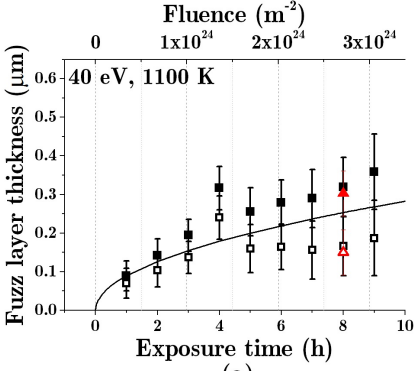

(a)

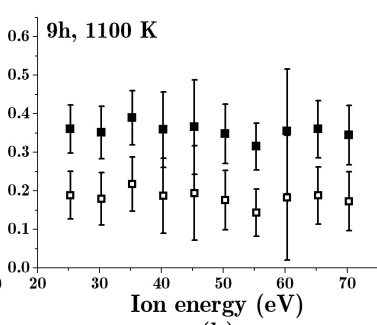

(b)

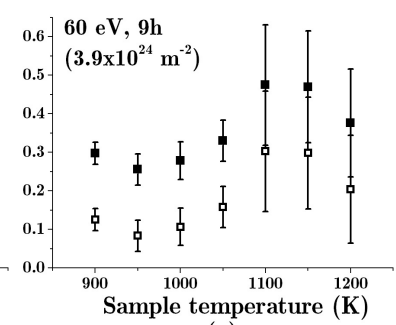

(c)

Figure 6: Measurements of the fuzz layer thickness using the FIB cross-section investigation for (a) the time sweep, showing the equivalent fluence on the top axis, (b) the ion energy sweep, and (c) the sample temperature. The vertical scale is the same in each figure. For each figure the conditions kept constant are displayed in the top left. The filled-in squares represent the measurements from the FIB, whereas the open squares have the deposition subtracted from the measurement. The red triangle in (a) represents the $4+4 \mathrm{~h}$ exposure (see section 4.5). The line shown in (a) is the fit of $x=(C \Phi)^{1 / 2}$ with $C=2.36 \times 10^{-38} \mathrm{~m}^{4}[2]$.

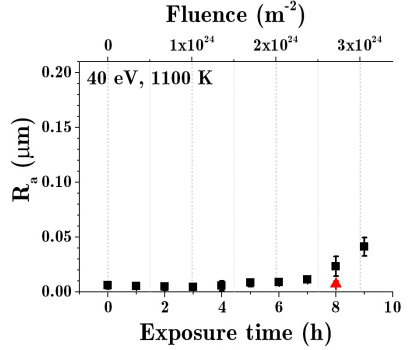

(a)

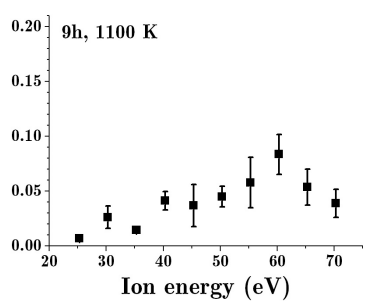

(b)

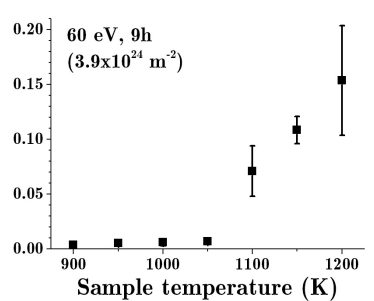

(c)

Figure 7: The roughness values shown for each parameter sweep. The vertical scale is the same in each figure. In (a) is the time sweep, showing the fluence on the top axis. In (b) is the energy sweep, and in (c) the temperature sweep. For each sweep, the conditions kept constant are displayed in the top left. The red triangle in (a) represents the $4+4 \mathrm{~h}$ exposure (see section 4.5. 


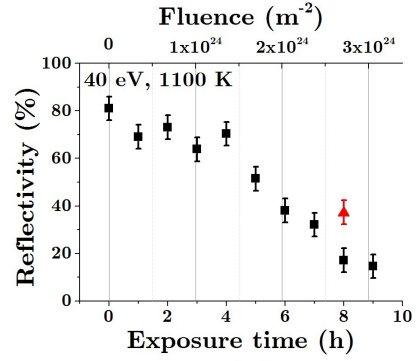

(a)

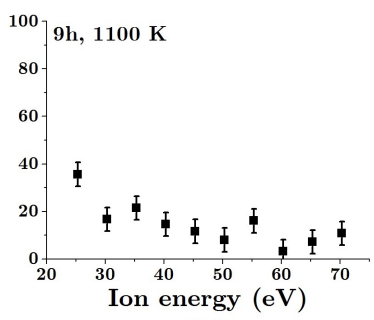

(b)

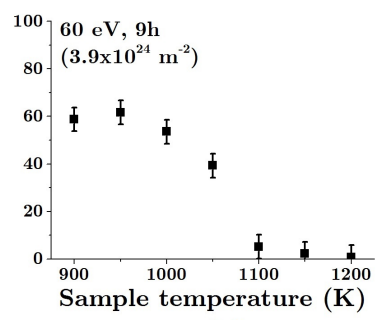

(c)

Figure 8: The reflectivity values shown for each parameter sweep. The vertical scale is the same in each figure. In (a) is the time sweep, showing the fluence on the top axis. In (b) is the energy sweep, and in (c) the temperature sweep. For each sweep, the conditions kept constant are displayed in the top left. The red triangle in (a) represents the $4+4 \mathrm{~h}$ exposure (see section 4.5).

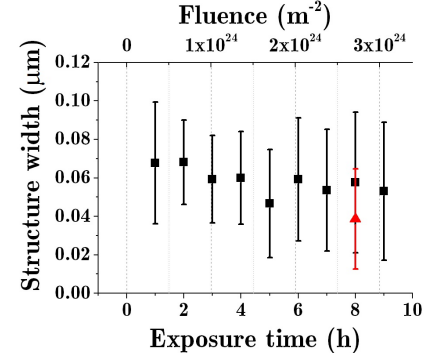

(a)

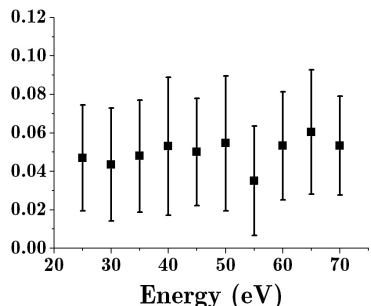

(b)

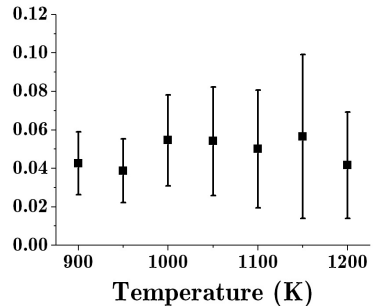

(c)

Figure 9: Measurements of the structure widths for the various parameter sweeps, with (a) showing the time (and hence fluence sweep), (b) the energy sweep, and (c) the temperature sweep. The red triangle in (a) represents the $4+4 \mathrm{~h}$ exposure (see section 4.5 . 


\section{Discussion}

175

\subsection{Boundary conditions for fuzz formation}

The boundary conditions for fuzz to form have been studied before in the literature. In terms of fluence, a minimum fluence has been hinted at in experiments, with Kajita et al. proposing a minimum fluence of $4 \times 10^{24} \mathrm{~m}^{-2}$ [1], and Petty et al. proposing an incubation fluence of $2.5 \times 10^{24} \mathrm{~m}^{-2}[2$. Regarding the samples presented in the fluence sweep of figure 2, the issue of defining when fuzz exists and when it does not is now apparent. There is no strict definition of fuzz, yet authors tend to agree that it is a tendril/coral/branch-like formation with random orientation.

An attempt is made to define a 'pre-fuzz', as opposed to regular/fully-formed fuzz, commonly seen at fluences $>10^{25} \mathrm{~m}^{-2}$. As fuzz is usually described with random orientation, pre-fuzz could be defined as having non-random orientation, i.e. showing dependence on the underlying grain direction. Such grain preference at low fluences has been seen before by several authors [27, 18, 28, 29]. This feature is presumably due to the tendrils growing upwards from the surface and beginning to bend in random directions, as can be seen in fig. 4 f for example. Hence at higher fluences the original orientation is lost. The second definition of fuzz is the tendril-like structure, therefore a fitting definition for pre-fuzz would be to not exhibit tendril-like structure.

Using these two definitions of pre-fuzz, one can make claims about boundary conditions from when pre-fuzz becomes fuzz. Looking first to fluence, as shown in fig. 2p, a preferred orientation is still observable at $2.0 \times 10^{24} \mathrm{~m}^{-2}$, this is more clearly seen at lower magnifications (provided in figure 10. However, this is no longer observed at $2.4 \times 10^{24} \mathrm{~m}^{-2}$. A trend is more apparent in the roughness value, as can be seen in figure $7 \mathrm{a}$, with a noticeable increase from $7 \mathrm{~h}$ to $8 \mathrm{~h}$. These hint at a cross-over point between 6 - $8 \mathrm{~h}$, at $2.4 \pm 0.4 \times 10^{24} \mathrm{~m}^{-2}$. The minimum fluence prediction by Kajita et al. of $4 \times 10^{24} \mathrm{~m}^{-2}$ is an extrapolation from thicker fuzz samples [1, and is not far off the value presented here. The incubation fluence, as predicted in recent work [2, was given at $2.5 \pm 1.5 \times 10^{24}$ 
$\mathrm{m}^{-2}$, which fits closely with the defined cross-over point from pre-fuzz to fuzz.

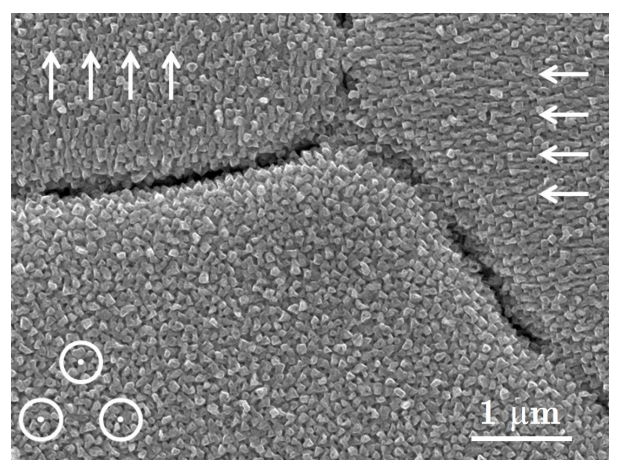

Figure 10: A lower magnification (x20,000) of the $6 \mathrm{~h}$ exposure also shown in fig 2b. The scale bar represents $1 \mu \mathrm{m}$. The observed orientation is shown with the arrows and circles.

Looking to the energy sweep, one could certainly argue that globules exist at $25 \mathrm{eV}$, whereas at $30 \mathrm{eV}$ tendrils exist. However, due to the previously mentioned fluence dependence by-product, this should not be taken as a fuzz formation condition. Previous work in the literature has noted a range of minimum energies for fuzz to form, from $12-27 \mathrm{eV}$ [3, 4, 5]. The SEM images of the energy sweep shown here do not present any apparent cross-over point, this is most likely at $\leq 25 \mathrm{eV}$, as other authors have reported.

Regarding the temperature, it is evident already from the SEM images that pre-fuzz becomes fuzz between 1050 and $1100 \mathrm{~K}$. This is also apparent in figures 75 and 8c where the roughness and the reflectivity drastically change from 1050 ${ }_{215} \mathrm{~K}$ to $1100 \mathrm{~K}$. The error on the temperature readings in the present experiments were due to the difference in emissivity between samples, and was deemed to be a maximum of $30 \mathrm{~K}$. Therefore, the widest gap possible is 1020-1130 K, giving the boundary condition for temperature at $1080 \pm 60 \mathrm{~K}$ (rounded to the nearest $10 \mathrm{~K})$. Sakaguchi et al. in [6] showed a minimum temperature of $1020 \pm 120 \mathrm{~K}$. Kajita et al.'s compilation chart in [5] shows $1000 \pm 100$ K. Recently Miyamato showed no fuzz formation even at $973 \mathrm{~K}$, giving a lower limit on fuzz formation [7. The different ranges have been presented diagrammatically in figure 11. It must be noted, that for the temperature ranges from the literature, these were 
taken from noting the cross-over point from when fuzz did not occur to when it did, giving the cross-over temperature range. However, the values on their temperature readings did not have associated error bars, whereas the present values do. As such, the ranges by the other authors should be even wider.

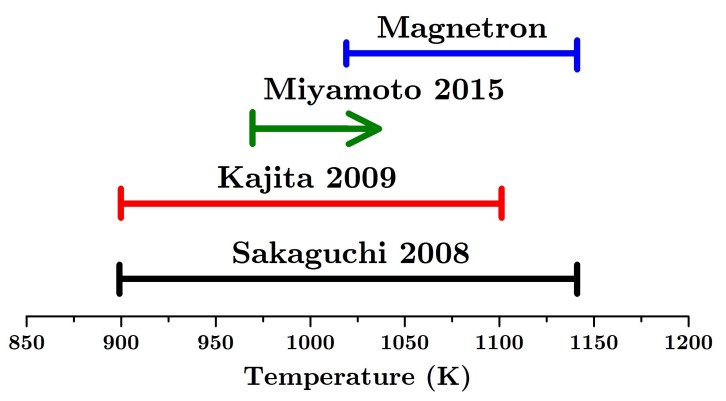

Figure 11: The various minimum temperatures for fuzz to form that have been thus far shown by Sakaguchi et al. in [6], by Kajita et al. in [5], by Miyamoto et al. in [7, and by the present results as shown in figure 4

\subsection{Competition of growth and annealing out of fuzz}

It has been shown by several authors that higher surface temperatures begin to anneal out the surface [30, 31, 32]. Annealing of fuzz has been reported to occur as low as $1300 \mathrm{~K}$ [32]. Perhaps this is also true at lower temperatures. There is evidently a competition of growth versus annealing out of the fuzz, which varies with the temperature. Several authors have cited a critical flux of $\sim 10^{21} \mathrm{~m}^{-2} \mathrm{~s}^{-1}$ for fuzz formation 33,31 . However, one could argue that at lower fluxes, the annealing dominates, and hence less fuzz is seen. As the present work indicates, fuzz can be made at lower fluxes, even an order of magnitude less, but they must be operated at lower temperatures, to reduce the effect of annealing.

Looking to the highest temperature sample here, shown in figure 5h, one can see that there is some apparent melting of the fuzz, as the tendrils are forming strange structures, and seemingly joining together, implying that the temperature is beginning to be detrimental to the fuzz growth. Samples getting thicker with higher temperatures have been observed in high flux experiments 
too [6, 27.

These results hint that annealing out could occur at even lower temperatures than currently reported. It is possible that some low level annealing out occurs even at $1200 \mathrm{~K}$, and maybe below, but this is only observable with low growth rates, as apparent in the present experiment.

\subsection{Comparison to PISCES-A}

Three samples were also exposed in the PISCES-A device based at UC San Diego, USA, at comparable fluences to the samples exposed in the magnetron device. Details of the experimental method for these samples are provided in 2]. The experimental conditions for these were a He ion energy of $75 \mathrm{eV}$, and a surface temperature of $1140 \pm 20 \mathrm{~K}$, the flux and fluence varied slightly between samples as is displayed in table 1. It is important to note the error on the time of exposure is $40 \mathrm{~s}$ [2]. Such short exposures lead to a significant error on the fluence. SEM images were taken of the samples, using the same methods as used for the magnetron samples. These images are shown in figure 12 .

\begin{tabular}{lllll}
\hline$\Phi$ & Time & $\Gamma$ & $\mathrm{T}$ & $\begin{array}{l}E_{\mathrm{i}} \\
(\mathrm{eV})\end{array}$ \\
\hline \hline $1.5 \pm 0.6$ & 107 & $1.4 \pm 0.04$ & $1140 \pm 20$ & 75 \\
$2.8 \pm 0.6$ & 200 & $1.4 \pm 0.04$ & $1140 \pm 20$ & 75 \\
$4.7 \pm 0.5$ & 286 & $1.7 \pm 0.4$ & $1140 \pm 20$ & 75
\end{tabular}

Table 1: Plasma conditions for the PISCES-A samples at low fluence.

Looking at the SEM images of the PISCES-A samples there are some subtle differences that set them apart from the samples exposed in a magnetron. In PISCES-A the structure, not the orientation, is uniform, with very clear tendrils appearing and no sign of globules. These would certainly all be defined as fuzz, rather than pre-fuzz. In the magnetron, there is a wide range of structures sizes. However, the tendrils seen in PISCES-A are much smaller than those seen in any magnetron sample. These were measured to be $\sim 28 \mathrm{~nm}$, as opposed to $\sim 50$ $\mathrm{nm}$ in a magnetron (see section 4.7). 


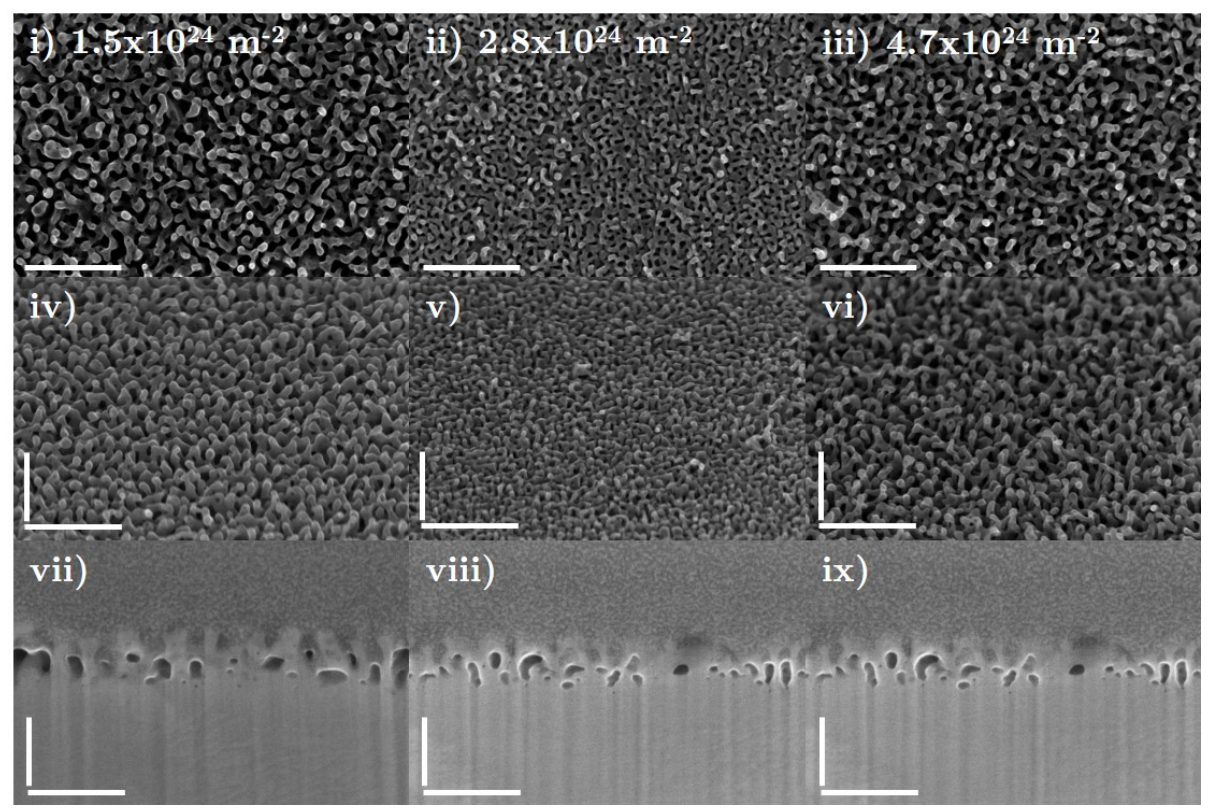

Figure 12: SEM images of low fluence samples exposed in PISCES-A for comparison to the samples exposed in a magnetron. Each column is a different sample, with the fluence noted in the top row. The top row is surface images, the middle row is surface images taken at a tilt of $45^{\circ}$, and the bottom row is the cross-section after FIB milling (also at $45^{\circ}$ ). A horizontal scale bar for each image in the bottom-left corner represents $500 \mathrm{~nm}$. In order to correct for the tilt in the bottom two rows, measurements must be multiplied by $1 / \sin \left(45^{\circ}\right)(=\sqrt{2})$; this has been accounted for by the vertical scale bars, representing $500 \mathrm{~nm}$ in the $y$-direction after accounting for tilt.

Regarding the existence of the tendrils in PISCES-A, this would lead one to think that this fuzz is more developed than that created in a magnetron. However, when looking at the thicknesses, a different picture shows. The PISCES-A samples were originally measured in 2], using the confocal microscope (CFM) technique. However, in order to be a fair comparison to the magnetron samples, these PISCES-A samples were measured again using the same FIB milling technique used for the present magnetron samples. The thicknesses of both devices are shown in figure 13 . The PISCES-A samples can be seen to be universally thinner than the magnetron samples, even when the deposition layer is subtracted. 


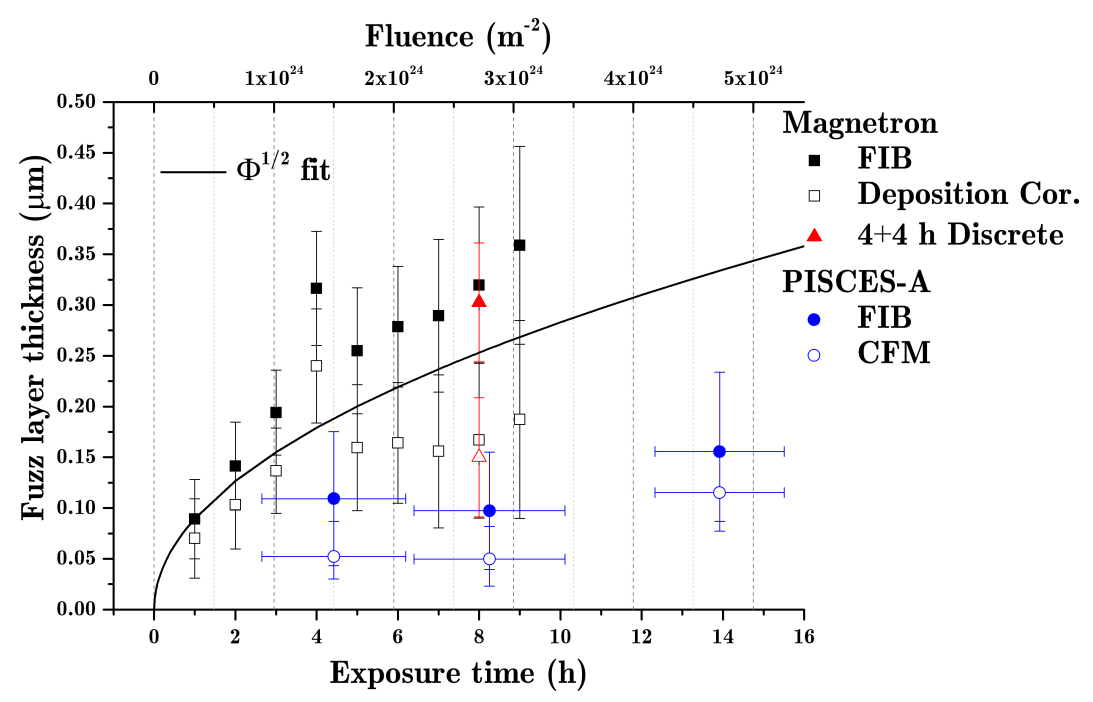

Figure 13: Thicknesses of the magnetron samples compared to those exposed in PISCES-A. The bottom axis is only relevant for the magnetron points, whereas the top axis is relevant for all devices. The magnetron measurements are represented by squares. The filled-in squares are the FIB measurements, the open squares are with the deposition rate accounted for. The red triangles are the discrete exposure, as discussed in section 4.5 . The blue circles are the samples exposed in PISCES-A, with the filled-in circles being the new FIB measurements, and the open circles the previous CFM measurements performed in [2].

It is quite plausible that the deposition in the magnetron causes the nonuniform structure. The samples grown in PISCES-A and in other devices in the literature do not have to contend with deposition. Depositing over the growing tendrils would occur at random intervals, and at random orientations to the growing tendrils, sometimes landing on the tops of the tendrils, other times on the sides.

As an aside note, the CFM and the FIB technique can be directly compared, as they have both been used to measure the PISCES-A samples. Both measurements are shown in figure 13 . The CFM technique used in [2] appears to slightly underestimate the FIB measurements by $\sim 50 \mathrm{~nm}$. This is most likely due to the scratching technique used. 


\subsection{Extension of the growth model}

The original fuzz growth model was given by Baldwin and Doerner in 9], and is easily converted into fluence as $x=(C \Phi)^{1 / 2}$, where $x$ is the fuzz layer time/fluence sweep plotted. It can be seen that the new measurements closely follow the original $\Phi^{1 / 2}$ fit.

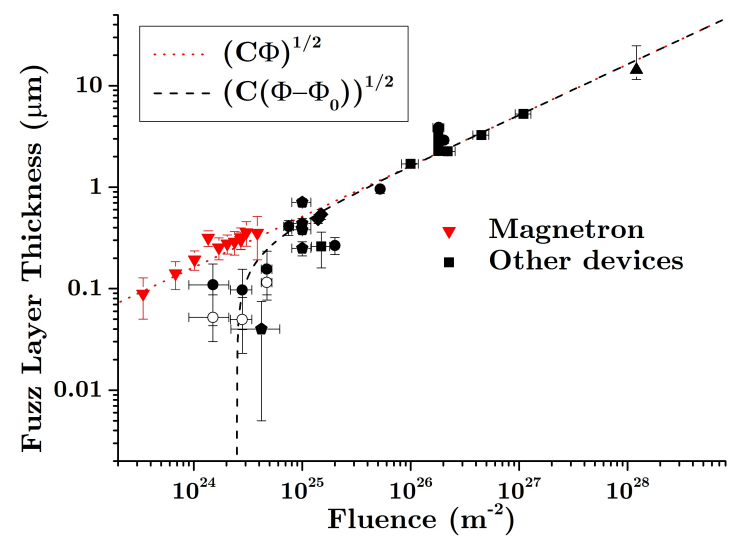

Figure 14: Thicknesses of fuzz created in other devices compared with the magnetron device. The compilation chart was originally created in [2]. The red triangles are the present magnetron samples, and the other symbols are all the other devices as discussed in [2]. The open circles represent the previous PISCES-A measurements using the CFM. The new measurements using the FIB are plotted slightly above these as full circles. Shown overlaid are two fits, the original $\Phi^{1 / 2}$ fit with a red dotted line, and the proposed fit of [2] that includes incubation fluence shown in the black dashed line.

Originally, it was reported by Baldwin et al. that the growth of fuzz was shown to have a $t^{1 / 2}$ dependence [9], and later work hinted that there was an incubation time necessary for fuzz to grow 33]. Recent work compiled many 
different devices covering four orders of magnitude of fluxes, thus to be able to compare all the devices, the discussion was changed to be about fluence, rather than time [2]. It was noted that the fuzz followed a $\Phi^{1 / 2}$ dependence, as is shown in figure 14. However, for the low fluence samples, it appeared that they were sitting below the $\Phi^{1 / 2}$ fit. An incubation fluence was then proposed, that is required before the growth of fuzz can begin, which follows along the same lines as the incubation time proposed by Baldwin, but reconciles devices using different fluxes. However, the present samples exposed in the magnetron follow the original $\Phi^{1 / 2}$ relation, seemingly not requiring an incubation fluence.

Regarding the differences for the low fluence work $\left(\sim 1 \times 10^{24} \mathrm{~m}^{-2}\right)$ between PISCES-A and the present magnetron samples, they have the same fluence, yet the magnetron has long exposure times $\left(\sim 3 \times 10^{4} \mathrm{~s}\right)$ and low fluxes $\left(1 \times 10^{20}\right.$ $\left.\mathrm{m}^{-2} \mathrm{~s}^{-1}\right)$, whereas the PISCES-A has short exposure times $\left(3 \times 10^{2} \mathrm{~s}\right)$ and large fluxes $\left(1 \times 10^{22} \mathrm{~m}^{-2} \mathrm{~s}^{-1}\right)$. Recent work by Khan et al. 34] involved even higher fluences $\left(1-5 \times 10^{26} \mathrm{~m}^{-2}\right)$, with layer thicknesses sitting below the trend again. In these experiments, the fluxes were even higher $\left(\sim 1 \times 10^{23} \mathrm{~m}^{-2} \mathrm{~s}^{-1}\right)$, with exposure times of $\sim 1 \times 10^{3} \mathrm{~s}$. Therefore, perhaps the notion of an incubation time could be re-introduced. Given the hypothetical situation of a device with a flux of $3201 \times 10^{24} \mathrm{~m}^{-2} \mathrm{~s}^{-1}$, exposed for $1 \mathrm{~s}$, it is reasonable to expect that fuzz would not grow, as there is simply not enough time for the surface to develop and for all the mechanisms involved with fuzz growth to take effect (see [35] for possible mechanisms at play). This implies there is some inherent incubation time necessary for fuzz to grow. Regarding the results of figure 14, it seems that the magnetron, with its long exposure times, easily surpasses the incubation time, whereas PISCES-A does not, and is playing catch-up with the $\Phi^{1 / 2}$ trend. The recent work by Khan et al. also suggest that given longer exposures the results would also follow the $\Phi^{1 / 2}$ trend.

As samples are exposed to higher fluences, the growth rate will decrease, as 330 it follows a $\Phi^{1 / 2}$ relation. However, for samples grown in a magnetron device, the deposition rate will remain constant. Therefore, at higher fluences, the thickness would be proportional to $\Phi$ rather than $\Phi^{1 / 2}$. This should certainly 
be investigated, since if the depositing $\mathrm{W}$ atoms really do integrate with the fuzz, then this would imply thicker samples (for the same fluence) than for LPDs. However, this would take significantly longer, as a fluence of $10^{25} \mathrm{~m}^{-2}$ in the current magnetron would take $\sim 28 \mathrm{~h}$.

\subsection{Discrete exposure time}

To date there has not been any study on whether a fuzzy sample created in one continuous exposure is comparable to one created in multiple runs. Discrete exposures have already been done before for very long fluence samples [2]. But should any precaution be taken with these samples? In the present work, a separate sample was prepared in the same manner as all of the others, then exposed for $4 \mathrm{~h}$, at which point the plasma and the heating were simultaneously switched off and the exposure time paused. The sample was left in the vacuum chamber overnight without breaking the vacuum. The following day the sample was treated as if new, and using the exact same procedure used before the sample was exposed for another $4 \mathrm{~h}$. The other conditions for the exposure were exactly the same as the $8 \mathrm{~h}$ exposure in the time sweep of figure 21. Thus they were both at $1100 \mathrm{~K}$, and with $40 \mathrm{eV}$ He ions. The two samples to compare are shown in the SEM pictures of fig. 2 and k. Higher quality SEM images are presented in figure 15 The thickness of the discrete sample was measured along with the other samples and is shown by the red triangle in figure 6 a. The roughness of the surface, the reflectivity, and the structure widths have also been measured and are presented in figures $7.9 \mathrm{a}$, respectively, represented by 355 the red triangles in each case. 


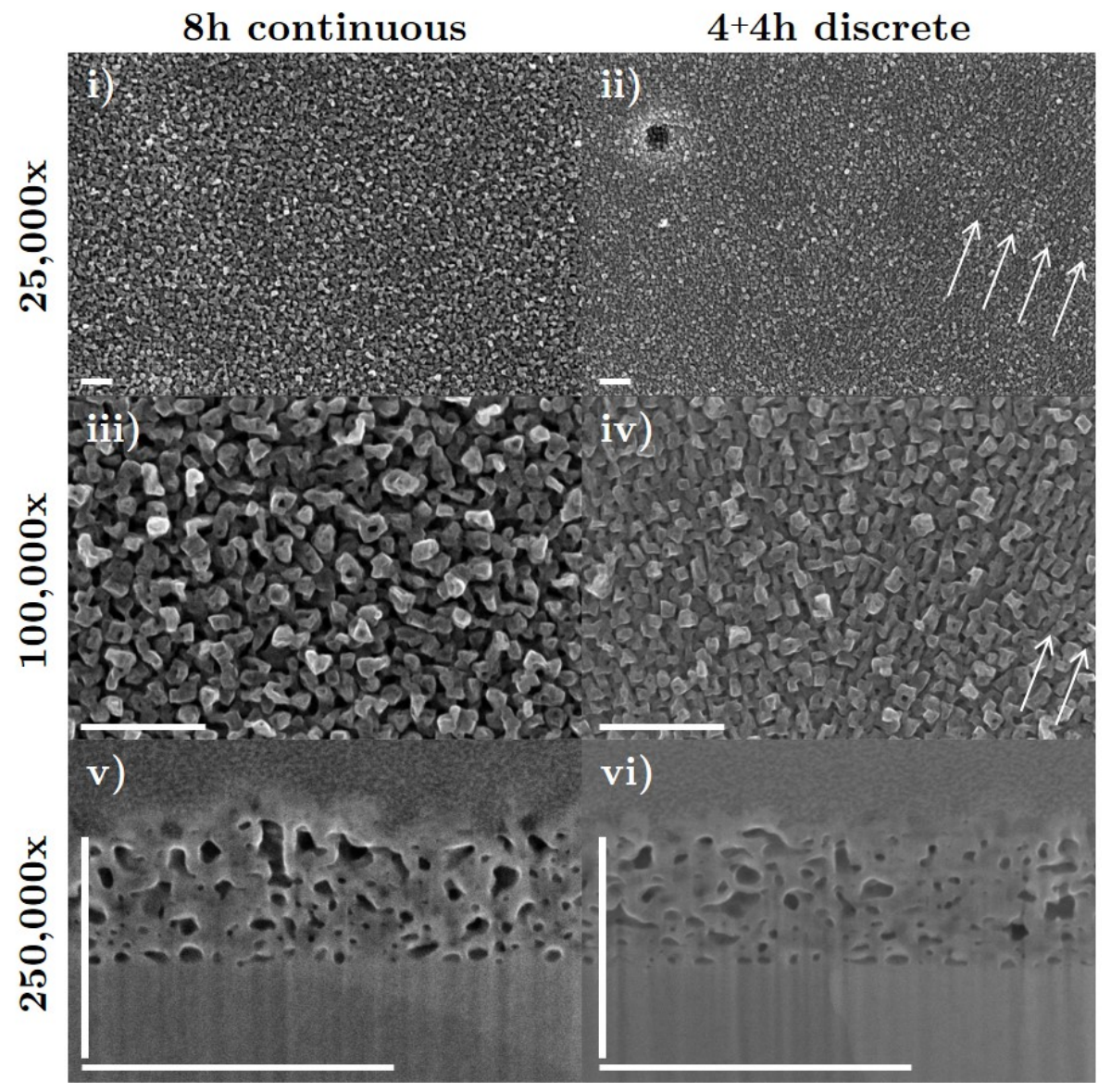

Figure 15: SEM images showing the comparison between continuous and discrete fuzz growth. Both samples were exposed for a total fluence of $2.7 \times 10^{24} \mathrm{~m}^{-2}$. The images on the left are samples exposed in one continuous $8 \mathrm{~h}$ exposure, whereas the images on the right underwent two $4 \mathrm{~h}$ exposures, left in vacuum overnight in between. The top row shows surface images taken at $25,000 \times$ magnification, the middle row is taken at $100,000 \times$, whereas the bottom row is the cross-section after FIB milling, and is taken at 250,000 $\times$ magnification. A scale bar is provided in the bottom left of each image, representing $500 \mathrm{~nm}$ in each case. As the bottom two images were taken at a tilt of $45^{\circ}$, any measurement in the $y$-direction must be multiplied by $1 / \sin \left(45^{\circ}\right)(=\sqrt{2})$. This correction factor is portrayed by a vertical line, representing 500 $\mathrm{nm}$ after accounting for the tilt. 
By comparing the two samples side by side, some interesting features can be seen. The SEM surface images, the roughness values, and the reflectivity values, all would suggest that the discrete sample is not as fully developed as the continuous sample, sitting more in line with lower fluence samples. However, 
the amount left over (in white), i.e. of fuzz grown due to the He. In this figure, it seems that after subtracting the thickness contributed by the depositing $\mathrm{W}$ flux, the amount left over for the discrete exposure is much more in line with the amount expected for the one-off $4 \mathrm{~h}$ exposure. It should be noted that looking to the thickness measurements of figure 6a, it is apparent that the actual $4 \mathrm{~h}$ exposure is sitting off the trend and is likely erroneous, and as such the trend has been interpolated to where it is expected to be. Though more samples are needed to verify whether this could be the case or not.

The annealing out of the fuzz has been hinted to be possible at lower temperatures than previously reported (see section 4.2), it is plausible that annealing out could occur before the exposure begins due to the nature of the experimental method. The present samples were heated to the required temperature over $\sim 15$ minutes, then the plasma was ignited and ramped up in power over 3 minutes before the bias on the sample was turned on, which is where the present samples were deemed to begin their exposure. During this time, the sample temperature was $>900 \mathrm{~K}$ for $\sim 8$ minutes before exposure began, in which some He could escape, and also some annealing may have occurred.

To shed further light on this, a dedicated annealing study should be done to fully understand if this could have been the case. One could surmise that there is cause to suspect differences may occur operating in discrete exposures, however, for high flux devices, with high fluence exposures, these differences are probably negligible.

\subsection{Reflectivity and roughness}

The reflectivity measurements presented here are comparable to reflectivity measurements performed in the literature. Sakaguchi et al. in [6] looked at the reflectivity of $\mathrm{W}$ samples during the growth of fuzz using a He-Ne laser at $632.8 \mathrm{~nm}$, hence $632.8 \mathrm{~nm}$ was chosen as the wavelength in figure 8 . They show that the reduction in reflectivity is greater at higher surface temperatures, as is also apparent here. They also show that the reflectivity of fully formed fuzzy surfaces is $\lesssim 1 \%$. Further work by Sakaguchi et al. reported also seeing that 


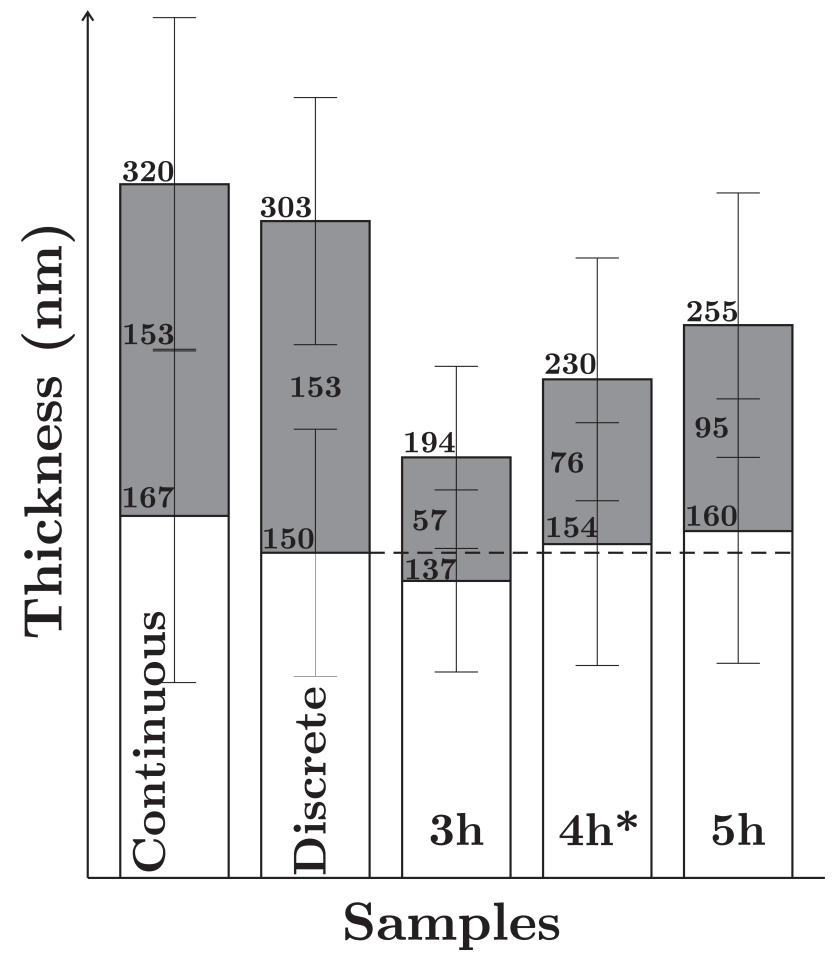

Figure 16: Comparison of the thicknesses of several samples, with the amount possibly caused by deposition highlighted on the top in grey, and the part not caused by deposition on the bottom in white. ${ }^{*}$ The values for the $4 \mathrm{~h}$ exposure were interpolated from the trend of figure 6. rather than the measured value.

the reflectivity is reduced more at lower wavelengths than at higher ones [40. Work by Kajita et al. also reports on the reflectivity decreasing with increasing fluences to nearly $1 \%[5]$.

The roughness values presented here in figure 7 are in line with values of roughnesses measured by other authors. Kajita et al. mentioned the roughness of fuzzy samples was measured to be $\sim 100 \mathrm{~nm}$. Their samples being much thicker and more developed than the present samples, this fits well with the rougher surfaces measured here, for example at $1200 \mathrm{~K}$ at $\sim 160 \mathrm{~nm}$ (see fig. 425 7 ). 


\subsection{Structure width}

Regarding the structure widths, as shown in 9 it can be seen that across all three parameter sweeps, there is very little change. The samples are well within each others' error bars, showing that there is a very wide variety in each sample. In the fluence sweep there is a potential decrease with increasing fluence, which is also seen in the work of Kajita et al. in [1]. At $6 \times 10^{24} \mathrm{~m}^{-2}$ they measure the widths to be $110 \pm 30 \mathrm{~nm}$, decreasing to $47 \pm 7 \mathrm{~nm}$ by $2.4 \times 10^{25} \mathrm{~m}^{-2}$. Whereas within this study, the widths of the structures only just reached $100 \mathrm{~nm}$ at the maximum error bar in a few cases, with a width of $53 \pm 36 \mathrm{~nm}$ for a fluence of

${ }_{435} 3 \times 10^{24} \mathrm{~m}^{-2}$. This difference could be due to the work of Kajita et al. being at much higher temperatures, partially annealing the structures, and hence them coming together to form larger structures at lower fluences. Averaging all the measurements from the energy and temperature sweep (which present no apparent trend), gives an average structure width of $50 \pm 31 \mathrm{~nm}$.

\section{Conclusions}

Three parameter sweeps have been performed in a magnetron device; the He ion fluence to the sample, the He ion energy, and the sample surface temperature. A cross-over point from pre-fuzz to fully formed fuzz has been found in section 4.1, with a fluence of $2.4 \pm 0.4 \times 10^{24} \mathrm{~m}^{-2}$, closely overlapping with

445 the incubation fluence discovered in previous work at [2] at $2.5 \pm 1.5 \times 10^{24} \mathrm{~m}^{-2}$. There has been no apparent cross-over point in the energy range from 25-70 $\mathrm{eV}$. Regarding the temperature, a clear cross-over point is seen at $1080 \pm 60 \mathrm{~K}$. From the parameter sweeps it was found that the best conditions for making fuzz in magnetrons is a fluence of $\geq 2.4 \times 10^{24} \mathrm{~m}^{-2}$, a He ion energy of $60 \mathrm{eV}$, and a surface temperature of $1100 \mathrm{~K}$. The competition between annealing-out and growth of fuzz has been discussed in section 4.2, hinting that annealing could occur at lower temperatures $(\sim 1200 \mathrm{~K})$ than previously seen $(\geq 1300 \mathrm{~K})$, presenting a potential issue for high-temperature low-flux experiments. 
The fuzz created in the magnetron is compared to low fluence experiments ues in section 4.6. The structure widths of the samples exposed in the magnetron are measured, showing a small decrease with the fluence, however, no apparent trend in the energy or temperature sweeps was observed. The average structure width was measured to be $50 \pm 31 \mathrm{~nm}$.

\section{Acknowledgements}

The authors would like to thank Alan Roby for his practical work involved in this experiment, David Sawtell for his help with the reflectivity measurements, and Matthew Baldwin for assistance with the PISCES-A samples. The authors would also like to thank the reviewers for their useful comments. TJ Petty would also like to gratefully acknowledge the University of Liverpool for his studentship, supported by the Engineering and Physical Sciences Research Council (EPSRC) doctoral training grant (Fusion DTC). 


\section{References}

[1] S. Kajita, N. Yoshida, R. Yoshihara, N. Ohno, M. Yamagiwa, TEM observation of the growth process of helium nanobubbles on tungsten: Nanostructure formation mechanism, Journal of Nuclear Materials 418 (2011) $152-158$.

[2] T. J. Petty, M. Baldwin, M. Hasan, R. Doerner, J. Bradley, Tungsten 'fuzz' growth re-examined: the dependence on ion fluence in non-erosive and erosive helium plasma, Nuclear Fusion 55 (2015) 093033.

[3] S. Takamura, N. Ohno, D. Nishijima, S. Kajita, Formation of Nanostructured Tungsten with Arborescent Shape due to Helium Plasma Irradiation, Plasma and Fusion Research 1 (2006) 051.

[4] M. Baldwin, T. Lynch, R. Doerner, J. Yu, Nanostructure formation on tungsten exposed to low-pressure rf helium plasmas: A study of ion energy threshold and early stage growth, Journal of Nuclear Materials 415 (2011) S104-S107.

[5] S. Kajita, W. Sakaguchi, N. Ohno, N. Yoshida, T. Saeki, Formation process of tungsten nanostructure by the exposure to helium plasma under fusion relevant plasma conditions, Nuclear Fusion 49 (2009) 095005.

[6] W. Sakaguchi, S. Kajita, N. Ohno, M. Takagi, H. Kurishita, Formation condition of fiberform nanostructured tungsten by helium plasma exposure, in: Proceedings of ITC18, pp. 246-249.

[7] M. Miyamoto, S. Mikami, H. Nagashima, N. Iijima, D. Nishijima, R. Dogation of the formation behavior of helium bubbles in tungsten, Journal of Nuclear Materials 463 (2015) 333-336.

[8] R. Pitts, S. Carpentier, F. Escourbiac, T. Hirai, V. Komarov, S. Lisgo, A. Kukushkin, A. Loarte, M. Merola, A. Sashala Naik, R. Mitteau, M. Sug- 
ihara, B. Bazylev, P. Stangeby, A full tungsten divertor for ITER: Physics issues and design status, Journal of Nuclear Materials 438 (2013) S48-S56.

[9] M. Baldwin, R. Doerner, Helium induced nanoscopic morphology on tungsten under fusion relevant plasma conditions, Nuclear Fusion 48 (2008) 035001 .

[10] G. Federici, C. Skinner, J. Brooks, Plasma-material interactions in current tokamaks and their implications for next step fusion reactors, Nuclear Fusion 41 (2001) 1967.

[11] Y. Ueda, J. W. Coenen, G. De Temmerman, R. P. Doerner, J. Linke, V. Philipps, E. Tsitrone, Research status and issues of tungsten plasma facing materials for ITER and beyond, Fusion Engineering and Design 89 (2014) 901-906.

[12] J. Brooks, R. Causey, G. Federici, D. Ruzic, Assessment of erosion and surface tritium inventory issues for the ITER divertor, Journal of Nuclear Materials 241-243 (1997) 294-298.

${ }_{525}$ [13] J. Brooks, J. Allain, R. Doerner, A. Hassanein, R. Nygren, T. Rognlien, D. Whyte, Plasmasurface interaction issues of an all-metal ITER, Nuclear Fusion 49 (2009) 035007.

[14] M. De Respinis, G. De Temmerman, I. Tanyeli, M. C. M. Van De Sanden, R. P. Doerner, M. J. Baldwin, R. Van De Krol, Efficient plasma route to nanostructure materials: case study on the use of m-WO3 for solar water splitting., ACS applied materials \& interfaces 5 (2013) 7621-7625.

[15] S. Kajita, T. Yoshida, D. Kitaoka, R. Etoh, M. Yajima, N. Ohno, H. Yoshida, N. Yoshida, Y. Terao, Helium plasma implantation on metals: Nanostructure formation and visible-light photocatalytic response, Journal of Applied Physics 113 (2013) 134301. 
[16] S. Kajita, T. Saeki, N. Yoshida, N. Ohno, A. Iwamae, Nanostructured Black Metal: Novel Fabrication Method by Use of Self-Growing Helium Bubbles, Applied Physics Express 3 (2010) 085204.

[17] S. Iyyakkunnel, L. Marot, B. Eren, R. Steiner, L. Moser, D. Mathys, M. Düggelin, P. Chapon, E. Meyer, Morphological changes of tungsten surfaces by low-flux helium plasma treatment and helium incorporation via magnetron sputtering, ACS Applied Materials and Interfaces 6 (2014) 11609-11616.

[18] O. El-Atwani, K. Hattar, J. Hinks, G. Greaves, S. Harilal, A. Hassanein, Helium bubble formation in ultrafine and nanocrystalline tungsten under different extreme conditions, Journal of Nuclear Materials 458 (2015) 216223.

[19] K. Woller, D. Whyte, G. Wright, Dynamic measurement of the helium concentration of evolving tungsten nanostructures using Elastic Recoil Detection during plasma exposure, Journal of Nuclear Materials 463 (2015) 289-293.

[20] R. Nygren, R. Raffray, D. Whyte, M. Urickson, M. Baldwin, L. Snead, Making tungsten work - ICFRM-14 session T26 paper 501 Nygren et al. making tungsten work, Journal of Nuclear Materials 417 (2011) 451-456.

[21] Y. Igitkhanov, B. Bazylev, R. Fetzer, The quantification of the key physics parameters for the DEMO fusion power reactor and analysis of the reactor relevant physics issues, KIT Scientific Publishing, 2015.

[22] T. J. Petty, J. W. Bradley, Tungsten nanostructure formation in a magnetron sputtering device, Journal of Nuclear Materials 453 (2014) 320-322.

560 [23] P. Virostko, M. Tichý, Z. Hunicka, P. Adámek, Measuring the Ion Current to the Substrate During Deposition of Thin Films by Hollow Cathode Plasma Jet, in: WDS'07 Proceedings of Contributed Papers, Part II, pp. 212-217. 
[24] Y. Pauleau, P. Barna, Protective Coatings and Thin Films: Synthesis, Characterization, and Applications, NATO ASI series 3, Springer Netherlands, 1996.

[25] W. Eckstein, Calculated Sputtering, Reflection and Range Values, MaxPlanck-Institut für Plasmaphysik, 2002.

[26] R. L. Merlino, Understanding Langmuir probe current-voltage characteristics, American Journal of Physics 75 (2007) 1078.

[27] G. De Temmerman, K. Bystrov, J. J. Zielinski, M. Balden, G. Matern, C. Arnas, L. Marot, Nanostructuring of molybdenum and tungsten surfaces by low-energy helium ions, Journal of Vacuum Science \& Technology A: Vacuum, Surfaces, and Films 30 (2012) 041306.

[28] H. Fan, Q. Yang, X. Li, W. Ni, J. Niu, D. Liu, Microscopic Damage of Tungsten and Molybdenum Exposed to Low-Energy Helium Ions, Plasma Science and Technology 17 (2015) 331-336.

[29] A. Khan, G. De Temmerman, T. W. Morgan, M. B. Ward, Effect of rhenium addition on tungsten fuzz formation in helium plasmas, Journal of Nuclear Materials 474 (2016) 99-104.

[30] M. Baldwin, R. Doerner, Formation of helium induced nanostructure fuzz on various tungsten grades, Journal of Nuclear Materials 404 (2010) 165173.

[31] S. Kajita, N. Ohno, M. Yajima, J. Kato, Growth annealing equilibrium of tungsten nanostructures by helium plasma irradiation in non-eroding regimes, Journal of Nuclear Materials 440 (2013) 55-62.

[32] F. W. Meyer, H. Hijazi, M. E. Bannister, K. A. Unocic, L. M. Garrison, C. M. Parish, Flux threshold measurements of He-ion beam induced nanofuzz formation on hot tungsten surfaces, presentation at the 15th PFMC conference in Aix-en-provence, France, 2015. 
[33] M. Baldwin, R. Doerner, D. Nishijima, K. Tokunaga, Y. Ueda, The effects of high fluence mixed-species (deuterium, helium, beryllium) plasma interactions with tungsten, Journal of Nuclear Materials 390-391 (2009) 886-890.

[34] A. Khan, T. W. Morgan, T. Petty, M. B. Ward, P. Mummery, Effect of Rhenium Concentration on Tungsten Fuzz Growth in Helium Plasmas at High Fluence, Submitted to Journal of Nuclear Materials on 05/05/2016 (2016).

[35] A. Ito, A. Takayama, Y. Oda, T. Tamura, R. Kobayashi, T. Hattori, S. Ogata, N. Ohno, S. Kajita, M. Yajima, Y. Noiri, Y. Yoshimoto, S. Saito, S. Takamura, T. Murashima, M. Miyamoto, H. Nakamura, Hybrid simulation research on formation mechanism of tungsten nanostructure induced by helium plasma irradiation, Journal of Nuclear Materials (2015).

[36] S. I. Krasheninnikov, Viscoelastic model of tungsten fuzz growth, Physica Scripta T145 (2011) 014040.

[37] Y. V. Martynenko, M. Y. Nagel, Model of fuzz formation on a tungsten surface, Plasma Physics Reports 38 (2012) 996-999.

[38] A. Lasa, S. K. Tähtinen, K. Nordlund, Loop punching and bubble rupture causing surface roughening - A model for W fuzz growth, EPL (Europhysics Letters) 105 (2014) 25002.

[39] M. Baldwin, R. Doerner, W. Wampler, D. Nishijima, T. Lynch, M. Miyamato, Effect of $\mathrm{He}$ on $\mathrm{D}$ retention in $\mathrm{W}$ exposed to low-energy, high-fluence (D, He, Ar) mixture plasmas, Nuclear Fusion 51 (2011) 103021.

[40] W. Sakaguchi, S. Kajita, N. Ohno, M. Takagi, In situ reflectivity of tungsten mirrors under helium plasma exposure, Journal of Nuclear Materials 390-391 (2009) 1149-1152. 